\title{
REVIEWER THANK YOU
}

\section{Wilderness E Environmental Medicine Peer Reviewers}

The editors express their sincere appreciation to the following individuals who completed peer reviews of articles submitted to Wilderness \& Environmental Medicine in 2021. WEM serves an important role in bringing literature to the scientific community. Our peer reviewers play an essential role in ensuring the merit and quality of the manuscripts we publish. Many of these individuals reviewed multiple papers, and some also serve on the editorial board and maintain section editor or associate editor duties.

(Notes: *WEM editorial board member; †WEM Reviewer-in-Training.)

Wendy Adams, USA

Subarna Adhikari, Nepal

William W. Aitken, USA

Billie Alba, USA

Sam Alfred, Australia

Kenton Anderson, USA

Michael Anderson, USA

Tracy Anderson-Dawson, USA $\dagger$

James Anholm, USA

Christa Arefieva, USA $\dagger$

Alok Atreya, Nepal

Chandrika Azad, India

Howard Backer, USA*

Santosh Baniya, Nepal

Buddha Basnyat, Nepal*

Jared Baylis, Canada $\dagger$

Cynthia M. Beall, USA

Jordan Max Benjamin, USA

Brad L. Bennett, USA*

Al M. Best, USA*

Sanjeeb Sudarshan Bhandari, Nepal $\dagger$

Aaron Robert Billin, USA

Cindy Carol Bitter, USA

Andrew Bivin, USA

Denise F. Blake, Australia

Erin Board, USA $\dagger$

Gemma Claire Lewis Bornick, USA

Benjamin Earl Brackett, USA $\dagger$

Karleigh Bradbury, USA

Marc Elliott Breen, USA

Robert Brickley, USA

Aaron Brillhart, USA

Joshua Briotti, Australia

Monika Maria Brodmann Maeder, Switzerland*

Emma Browne, UK
Hermann Brugger, Italy

William Bunzel, USA $\dagger$

Omer Lee Burnett, USA

Frank K. Butler, USA

Michael Cardwell, USA

Luis Carus, Spain

James L. Caruso, USA

Marc Cassone, USA

John Castellani, USA

John Robert Clarke, USA $\dagger$

Richard F. Clark, USA

Taryn Clark, USA

Jonathan Clemens, USA $\dagger$

Christanne H. Coffey, USA

Kirsten Coffman, USA

Geoffrey B. Comp, USA $\dagger$

Nicholas J. Connors, USA

Keith Conover, USA

Gregory A. Cummins, USA $\dagger$

Michael Dacre, USA

Daniel Danzl, USA

Gali Dar, Israel

Saibal Das, India

Hillary E. Davis, USA $\dagger$

Mark Davis, USA

Terri Davis, USA $\dagger$

Christopher Burney Davis, USA

Suvash Dawadi, Nepal

David DeGroot, USA

Thomas DeLoughery, USA

Petar J. Denoble, USA

Robert W. Derlet, USA

M. Di Nicola, Italy

James H. Diaz, USA

Paolo Dominelli, Canada

Jim Donovan, USA
Jennifer Dow, USA

John Downs, USA

Sebastian Drago, Chile

Caleb Dresser, USA

Kurt Power Eifling, USA

Brett Romano Ely, USA

Timothy B. Erickson, USA

Marika Falla, Italy

E.J. Favaloro, Australia

Preston J. Fedor, USA

Ariel J. Feifel, USA $\dagger$

Francesco Feletti, Italy

Arielle Filiberti, USA $\dagger$

Patrick Fink, USA

Justin Fleischer, USA $\dagger$

Joseph Derek Forrester, USA

Luanne Freer, USA

Katharine H. Ganly, UK†

Arun Ganti, USA $\dagger$

Gordon G. Giesbrecht, Canada

Todd Golden, USA

Torrey Lisa Goodman, USA

Audrey Gray, USA $\dagger$

Mike Greene, UK

Spencer Greene, USA

Peter Greenwald, USA

Justin Grisham, USA $\dagger$

Astrid Haaland, USA $\dagger$

Susan Haapaniemi, USA

Peter Hackett, USA*

Vidal Haddad Jr., Brazil

Lauren Hansen, USA

N. Stuart Harris, USA

Jamie Harshman, USA $\dagger$

Tatiana Havryliuk, USA

Seth C. Hawkins, USA 
Alexandra Herndon, USA $\dagger$

David Hillebrandt, UK

Ross Hofmeyr, South Africa

Susan R. Hopkins, USA

Kirsten Hornbeak, USA

William Patrick Howlett,

United Republic of Tanzania

Hillary R. Irons, USA

Arthur (Tony) Anthony Islas, USA

Bryan Jarrett, USA

Dominique Jean, France

David Jerome, Canada

Eric Johnson, USA

Kelsey Joyce, UK†

Lee A. Kaplan, USA

Tao Ke, USA $\dagger$

Eric Kelley, USA

Daniel Keyler, USA

Kenneth W. Kizer, USA*

Beat Franz Knechtle, Switzerland

Oliver Kreuzer, Switzerland

Jeff Lane, USA $\dagger$

Ricky Langley, USA

Stephanie Lareau, USA*

Lorie H. Laroche, Canada $\dagger$

Wolfgang Lederer, Austria

Stephen Y. Liang, USA

James Lieberman, USA

Grant S. Lipman, USA

Christoph Fabian Lutter, Germany

Sonia Mariano, USA $\dagger$

Paul Martin, USA $\dagger$

Cristina Martin Sierra, Spain

Dana Mathew, USA $\dagger$

Todd M. McGrath, USA

Scott Ellis McIntosh, USA

Jake Neil McRae, USA

Ben Meadley, Australia

Jesse Meik, USA

Paul Meriac, USA $\dagger$

Daniel Migliaccio, USA

Nathan Miller, USA $\dagger$

RJ Mills, USA $\dagger$

Seyed Mostafa Mirakbari, Islamic Republic of Iran
Matthew L. Moorman, USA $\dagger$

Dan Morgenstern-Kaplan, Mexico

Daniel S. Morris, UK

Audry Morrison, UK

Sandra Mara Brasileiro Mota, Brazil

Michael E. Mullins, USA

Martin Musi, USA $\dagger$

Bella Shah Nagappan, USA $\dagger$

Andrew T. Nathanson, USA

Maria Nemethy, USA

Matias Nochetto, USA

Rachel Nygaard, USA

Aebhric O'Kelly, Germany $\dagger$

Gerald O'Malley, USA

Alan Oram, USA

Edward Joseph Otten, USA*

Peter Paal, Austria

Deb Pandey, Nepal

Manjari Pandey, USA

Ryan Paterson, USA

Ziva Petrin, USA

Lara Phillips, USA

Walker Plash, USA

Thomas Platts-Mills, USA

Ross Pollock, UK

Matiram Pun, Nepal*

Robert H. Quinn, USA

Balram Rathish, India

Saumitra Rege, USA

Aaron Reilly, USA

Randal Reinertson, USA $\dagger$

Luisa Ricciardi, USA

Conal Roche, USA $\dagger$

George W. Rodway, USA

Jerome J. Rogich, USA $\dagger$

Steven Roy, Canada

Brent Ruby, USA

Anne-Michelle Ruha, USA

David A. Rust, USA

Roy Salgado, USA

Kathleen D. Saxon, USA

Volker Schöffl, Germany*

Sam Schimelpfenig, USA

Tod Schimelpfenig, USA
Andrew Schmidt, USA

Robert Schoene, USA

Walter Schrading, USA

Andreas Schweizer, Switzerland

Zina Semenovskaya, USA

Justin Sempsrott, USA

Alison Sheets, USA $\dagger$

Charley Shimanski, USA

Anjana Silva, Sri Lanka

Loïc Simon, France

Rodrigo Siqueira-Batista, Brazil

William R. Smith, USA

Inigo Soteras, Spain

Susanne J. Spano, USA

Jeffrey Spindel, USA $\dagger$

Peter St. Marie, USA

Rom Stevens, USA

Giacomo Strapazzon, Italy

Guenther Sumann, Austria

Choo Hock Tan, Malaysia

Colin Tilbury, South Africa

Martha Caperton Tissot Van Patot, USA*

Romain Torrents, France

Morgan Torris, USA $\dagger$

Jared Vagy, USA

Christopher Van Tilburg, USA

Scott R. Votey, USA

Matthias Walcher, Italy

David A. Warrell, UK

Brandon Warrick, USA

Julian White, Australia

Eranga Wijewickrama, Sri Lanka

Nicole Williams, USA

Bryan Wilson, USA

Matthew Wilson, USA

Kenneth Wofford, USA

Leo Wood, USA $\dagger$

Cedric M. Yoshimoto, USA

David Young, USA

Ken Zafren, USA*

Thomas R. Zilker, Germany

Benjamin Zweifel, Switzerland 